\title{
Simulation Method for Mission Reliability Assessment of Space Telemetry Tracking and Command System with Dynamic Redundancy
}

\author{
Haiyue $\mathrm{Yu}^{*}$ and Xiaoyue $\mathrm{Wu}$ \\ School of System Engineering, National University of Defense Technology, Changsha, 410073, China
}

\begin{abstract}
The mission reliability of space telemetry tracking and command (TT\&C) systems directly determines whether the space mission can be carried out successfully. In actual situations, some missions of TT\&C systems may just be required to be completed before a given time, rather than be performed in a specified time window. Taking advantage of this feature, mission failures in the originally arranged time period could then be re-executed later according to the rescheduling algorithm. The resources to be used and the time to be executed are two kinds of uncertainties that exist in rescheduling. This paper treats them as dynamic redundancy. The existing evaluation methods are insufficient to evaluate the mission reliability of system under this condition. This paper proposes a heuristic rule based on the system operation-principle to obtain the basic scheduling plan of the mission and then designs a reactive scheduling strategy to deal with mission failures during the execution process. Based on this scheduling architecture, a basic scheduling plan combined with a fault handling strategy can be obtained. A Monte Carlo method is used to simulate the components' failure and repair, and a discrete event simulation process is used to simulate the possible execution options during the execution process. A collection of altered execution options combined with the execution result of the mission can be gathered after a huge amount of simulation. The approximate mission reliability of the system can then be assessed through a statistic reference to this collection.
\end{abstract}

Keywords: mission reliability; dynamic redundancy; uncertainty scheduling

(Submitted on March 26, 2019; Revised on April 15, 2019; Accepted on June 17, 2019)

(C) 2019 Totem Publisher, Inc. All rights reserved.

\section{Introduction}

The space telemetry tracking and command (TT\&C) system is responsible for the tracking, telemetry, telecontrol, and communication missions of spacecraft and their payloads [1]. As the only link between spacecraft and the earth, space TT\&C systems participate in all phases from spacecraft launch to return. Therefore, the mission reliability of TT\&C systems is directly related to the successful implementation of the space mission.

The mission reliability of the space TT\&C system refers to the ability of the system to accomplish the required functions in each mission phase of the spacecraft. Whether the spacecraft is launching, in orbit, or in the return phase, the system mission can be classified into two categories according to its functions. One type is the TT\&C mission, which is in charge of spacecraft tracking, telemetry, and telecontrol. The other type is the communication mission, which carries out the payload data transmission and voice image communication (manned space) with the spacecraft [2]. These tasks can also be divided into two types according to their importance. Real time tasks are strict in time and must be completed in the scheduled time, such as tasks of the spacecraft in the launch phase. Non-real time tasks, especially the tasks in the orbit phase, do not need to be executed in a precise time, but require the task to be completed within a specified period of time. This paper considers that the non-real time tasks have a redundant backup in the executable time. In the actual scheduling, the system usually does not arrange a resource to the redundant time in advance. It reschedules the arrangement to utilize this redundant time after the mission fails. This rescheduling brings two kinds of uncertainty that cannot be known in advance: the restart time of the failure task and the resources to be used. In this paper, the uncertain backup in the system due to the redundant executable time of tasks is called dynamic redundancy. 
From the literature available, the existing mission reliability evaluation methods of the space TT\&C system mainly include some analytical methods, including the reliability block diagram, fault tree analysis, and Markov method, and some simulation methods. The reliability block diagram [3] and fault tree analysis [4] are two static evaluation methods that cannot assess the system dynamic behavior. With the application of fault tolerance, redundancy, and other techniques in the system to improve the system reliability, researchers have extended the two models to dynamic reliability diagrams and dynamic fault trees. These two extended models and the Markov method [5] can describe the dynamic behavior of system such as repairable, hot and warm backups, failure correlation, and sequence correlation. However, difficulties still exist in the process of modeling and obtaining solutions when the above methods are used to transform the uncertainty in complex systems into possible system structures or states. Xie et al. [6] carried out space TT\&C system mission reliability modeling and analysis work. They stated that the TT\&C devices on different geographic locations have different tracking times, and the reliability logical structure of the devices involved in the mission over a different period of time has changed. Thus, they pointed out that the space TT\&C system mission reliability model is a phased mission system (PMS) with variable structures. Wu et al. [7] also treated the space TT\&C system as a PMS and divided the phased mission according to the time point when TT\&C resources change. Then, they established the continuous-time Markov chain model of each phased mission and obtained the final result of whole system mission reliability. A PMS modeling method can simplify the modeling process; however, the existence of dynamic redundancy can make the relationship between various phases become more complex. There is still no proven method to solve this problem.

The simulation method is a commonly used method in engineering. Yang et al. [8] used the Petri net simulation method to analyze the reliability of TT\&C missions and statistically analyzed key TT\&C resources that would cause failures of missions. Due to the high reliability and short task window in aerospace engineering, the direct simulation method requires a large number of simulation times to yield an acceptable reliability simulation result. To this end, Xu [9] proposed a simulation based on the limited sampling method to evaluate the mission reliability of the space TT\&C system. In this paper, we use a simulation method to evaluate the reliability of the system with dynamic redundancy. Wu [10] designed a Monte Carlo simulation approach to estimate the mission reliability of a TT\&C system. The system was transferred to a PMS with time redundancy in a single phase. There are two simulation processes we can choose. The first process is simulation evaluation under a scheduling plan. A simulation is divided into two parts: the first part generates several feasible scheduling plans according to the scheduling algorithm, and the second part simulates the failure and maintenance of the equipment in each plan and then reschedules the failure mission. The second process is simulation evaluation without a scheduling plan. A simulation consists of only one part and considers the scheduling rules, equipment failure and maintenance, and fault handling methods together in the simulation process. This means that the simulation process handles the occurrence of mission failures and resource allocation simultaneously. The paper uses the first simulation process, which is more in line with the reality of real TT\&C process.

The problem about scheduling with uncertainty can mainly be divided into two parts: the first part involves the duration risk, which is the duration of each work that cannot be determined previously, and the second part involves the technique risk, which means that system malfunctions may occur during the mission process [11]. Then, the solution of these problems can be divided into two kinds: robust (proactive) scheduling and reactive scheduling [12]. The robust scheduling sets some redundancy structures (resource redundancy and time redundancy) to the baseline scheduling plan before the mission execution, and these redundancies can deal with the occurrence of uncertainty automatically during the mission process. Reactive scheduling deals with uncertainty using the reactive strategy. There is no redundancy in the baseline scheduling plan, and the plan is rescheduled according to the strategy as uncertainty occurs. This paper mainly considers the reactive scheduling strategy.

The remainder of this paper is organized as follows. Section 2 designs heuristic scheduling rules according to the system operation principle to complete the resource allocation. In Section 3, a rescheduling algorithm is proposed to reschedule the failure mission with dynamic redundancy. Section 4 presents a simulation scenario of the space TT\&C system and uses a Monte Carlo method combined with a discrete event simulation principle to assess system mission reliability. The paper is concluded in Section 5.

\section{Scheduling Problem Description and Heuristic Algorithm Design}

Spacecraft have a long running time in orbit with large demands for measurement, control, and communication tasks. A large number of TT\&C resources are used repeatedly, and researchers must consider their maintenance. For these reasons, researcher must design a resource scheduling algorithm to generate a scheduling plan to complete system missions. Current literature on scheduling problems mainly focuses on the scheduling optimization problem, which involves finding an optimal or better solution under the objective function. However, using the simulation method to evaluation system mission reliability, we must first find the feasible scheduling plans in the solution space as much as possible. Thus, without an 
objective function and optimization algorithm, this scheduling problem is different from the scheduling optimization problem. This section mainly includes two elements: a description of the scheduling problem and the heuristic algorithm design.

\subsection{Description of Scheduling Problem}

The space TT\&C system is a large-scale system that is composed of the space TT\&C center and its TT\&C stations. In this paper, we only consider two TT\&C station resources involved in scheduling: the ground station and the relay satellite. The actual undertaker of the TT\&C mission is the antenna, and each antenna belongs to a TT\&C station. The service objects of the system are the satellites. The visible relationship between the satellite and the antenna is the basis for the system to carry out resource scheduling. The satellite's attributes define its trajectory and motion state, and the ground station's properties describe its geographic location. Relay satellites are in a geostationary satellite orbit, but the right ascension of the ascending node (RAAN) is different. The characteristics of the ground station antenna include the elevation angle, azimuth angle, and cone angle. The above attributes determine the antenna and satellite visible relationship together.

Decision variables: The decision variables of the scheduling problem are the time interval when each antenna serves these satellites. A set of values for all decision variables constitutes a scheduling plan.

Constrains: During the process of determining whether a TT\&C station antenna is scheduled to serve a satellite, a set of domain constraints must be satisfied. In general, they consist of visible relationship limits, resource constraints, and mission requirements.

The visible relationship limits include the satellite-ground and inter-satellite visible relationship. They mainly describe the visible time window and the current operating status of the satellite (ascending/descending).

The resource constraints are formalized as follows: service types and operating requirements of each antenna. The service types in this paper are divided into TT\&C antenna, data transmission antenna, and function integrated antenna. In Section 1, we divide the TT\&C system mission into two types: TT\&C mission and communication mission. The essence of the communication mission is the downlink high-speed data transmission, which is completed by the data transmission antenna. The function integrated antenna can complete the two mission simultaneously and compensate for the shortage that the data transmission speed of the TT\&C antenna is insufficient for communication missions. The operating requirements of antennas mainly include antenna service preparation time and serve duration time. This paper predetermined that the ground station antenna takes five minutes to point to a new target, and its service time is the remaining time in the current satelliteground visible window. The relay satellite antenna preparation time is 30 minutes, and the service time is five minutes.

The mission requirements in this paper select the daily missions of the satellites. One satellite corresponds to a mission group that includes three index type: mission time index per lap, mission number index per day, and mission time index per day, as shown in Table 1. Different satellites have different mission priorities.

\begin{tabular}{|c|c|c|}
\hline \multirow{2}{*}{ Index type } & \multicolumn{2}{|r|}{ Mission } \\
\hline & ID & Name \\
\hline \multirow{2}{*}{$\begin{array}{l}\text { Mission time } \\
\text { index per lap }\end{array}$} & $\mathrm{a}$ & Minimum TT\&C service time per lap (minutes) \\
\hline & $\mathrm{b}$ & Minimum data transmission time per lap (minutes) \\
\hline \multirow{4}{*}{$\begin{array}{l}\text { Mission number } \\
\text { index per day }\end{array}$} & $\mathrm{c}$ & Data transmission number in ascending phase per day \\
\hline & $\mathrm{d}$ & TT\&C service number in ascending phase per day \\
\hline & $\mathrm{e}$ & Data transmission number in descending phase per day \\
\hline & $\mathrm{f}$ & TT\&C service number in descending phase per day \\
\hline \multirow{2}{*}{$\begin{array}{l}\text { Mission time } \\
\text { index per day }\end{array}$} & $\mathrm{g}$ & Minimum TT\&C service time per day (minutes) \\
\hline & $\mathrm{h}$ & Minimum data transmission time per day (minutes) \\
\hline
\end{tabular}

\subsection{Heuristic Scheduling Algorithm}

The algorithm introduced in this part is a combination of heuristic rules and the discrete event simulation process.

The heuristic rules are formalized as follows:

- Rule 1: Determine the resource allocation sequence according to the satellite mission priority, with high priority missions first. Missions with the same priorities define the priority by the mission's unsatisfied rate. Obtain a random selection if the unsatisfied rates are also the same. 
- Rule 2: Schedule the ground station resources first, and then schedule relay satellite resources if the mission is unsatisfied.

- Rule 3: Meet the mission time index per lap first, then the mission number index per day, and then the mission time index per day.

- Rule 4: Under the same mission index type, the service type that the mission needs is determined by the satisfied rate of the TT\&C mission and communication mission. Choose the type with the lower rate first.

- Rule 5: If several antennas can be allocated, choose the one with the lower using rate. Obtain a random selection if the using rates are also the same.

For the heuristic algorithm, the general process of simulation is shown as follows, and the resource heuristic scheduling algorithm for idle satellite is shown next. The general simulation processes is formalized as follows:

- Step 1: Set the simulation time, and then compute the visible relationship between each antenna and satellite. Set the simulation step, and perform the simulation step by step.

- Step 2: Each time the simulation enters into a new time slice, the resource's serving state is updated. The resources that complete the service by the current simulation time according to the schedule plan are released in time.

- Step 3: Pick up all the satellites that are not getting serviced in the current time. Sequence these idle satellites according to Rule 1, and then allocate the resources for them in order.

The resource heuristic scheduling algorithm describes the process of arranging an antenna to an idle satellite, as shown in Figure 1.

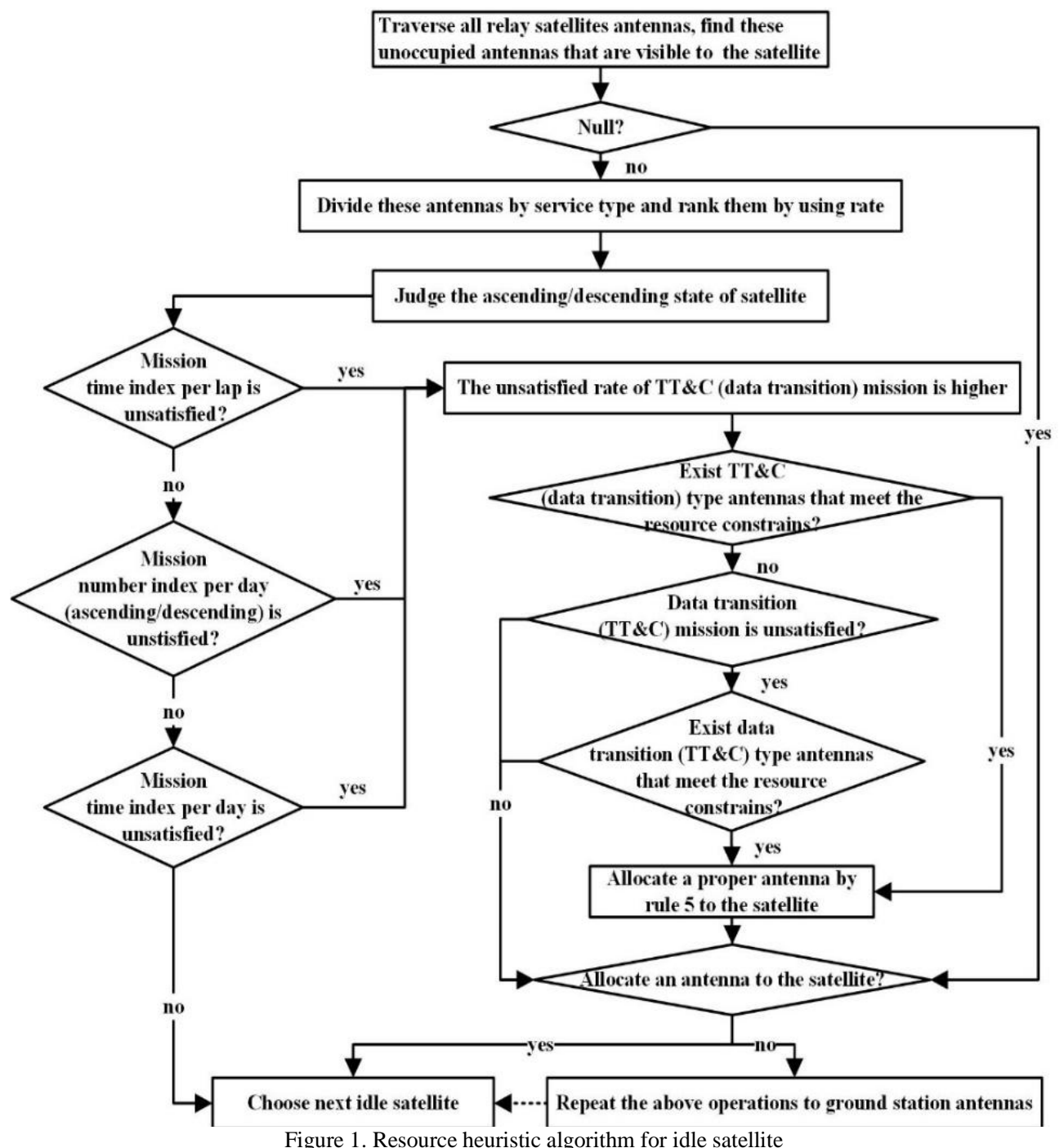

Figure 1. Resource heuristic algorithm for idle satellite 
- Step 1: Find the visible unoccupied antennas for the idle satellite from the visible relationship set in the current simulation time slice.

- Step 2: Compare each type of mission index according to Rule 3, and determine the index type that would allocate resources for it.

- Step 3: Choose the service type that the mission needs according to Rule 4 under the determined mission index.

- Step 4: Find the alternative antennas that satisfy the service type and operating requirements of the resource constraints described in Section 2.

- Step 5: Choose a proper antenna according to Rule 5 from these alternatives, and then allocate it to the satellite.

\section{Designation of Fault Reactive Strategy}

The strategy is designed for system missions with dynamic redundancy. Following this strategy, schedulers can reschedule the failure mission that is caused by resource malfunctions as long as it occurs during the system mission execution and the mission has dynamic redundancy. This section is composed of two parts: the fault react strategy and the rescheduling algorithm.

\subsection{Fault Reactive Strategy}

The strategy should re-execute the failure mission and minimize changes in the original scheduling plan. First, we give a definition of the failure mission insertable time interval: it describes the time interval that the failure mission may be inserted. The starting point of this interval is the earliest re-execution time of the failure mission, and the end point of this interval is the latest finish time of re-execution.

- Strategy 1: The inserted position can only be in the free visible time period, without replacing any of the scheduled tasks.

- Strategy 2: The failure mission insertable time interval is concerned with the mission index type that the failure mission belongs to. If the index is the mission time index per lap, then the time interval is defined as [failure occur time, end time of this lap]. If the index is the mission number/time index per day, then the time interval is defined as [failure occur time, end time of this day].

- Strategy 3: Choose the antenna with the lowest using rate when several antennas are available.

- Strategy 4: The mission is not rescheduled again if the service resource malfunctions again after rescheduling.

\subsection{Failure Mission Rescheduling Algorithm}

The algorithm introduced here is a combination of the fault react strategies and the discrete event simulation process.

- Step 1: On the basis of a given scheduling plan generated by the first part of simulation, carry out the second part simulation by the simulation step. Each time the simulation enters into a new time slice, judge whether the antennas have malfunctioned. If failures occur, determine the failure mission insertable time interval [M_earlist, M_latest] for each failure mission according to Strategy 2.

- Step 2: Traverse each visible relationship record [Fstart, Fend], find the time intervals [begin, end] that are visible to the failure mission satellite, and locate them in the failure mission insertable time interval [M_earlist, M_latest], as shown in the shadows of Figure 2.

- Step 3: Make another filter for the time intervals [begin, end], and remove the time periods for which the service has been arranged and the unusable time periods caused by antenna malfunction. Keep the time periods that are unoccupied and usable. The service type meets the mission demands, and the time span meets the operating requirements of the resource constraints.

- Step 4: Choose a time period from these alternative time periods according to Strategy 3, and arrange the failure task in this period.

\section{User Case for Mission Reliability Simulation}

This section includes two parts: (1) a module structure introduction of the simulation platform, and (2) a user case description and simulation result analysis.

\subsection{Simulation Platform}

The simulation platform is coded in Microsoft Visual Studio C\# and based on .Net framework 4. The input interface can 
accomplish the TT\&C scenario, constrain editing, and set the simulation parameters and fault parameters of each antenna. The simulation module is composed of two parts: resource scheduling and reliability simulation. The former generates different scheduling plans through several times of simulation under the resource heuristic scheduling algorithm, and the latter obtains the reliability simulation result of each scheduling plan. The TT\&C scenario can be saved as an XML document, and the scheduling plan and reliability simulation result are saved as an Excel file.

The simulation of antenna malfunction is performed by the Monte Carlo method according to the fault parameters. The reliability simulation result includes two parts: the failure mission record and the rescheduling plan.

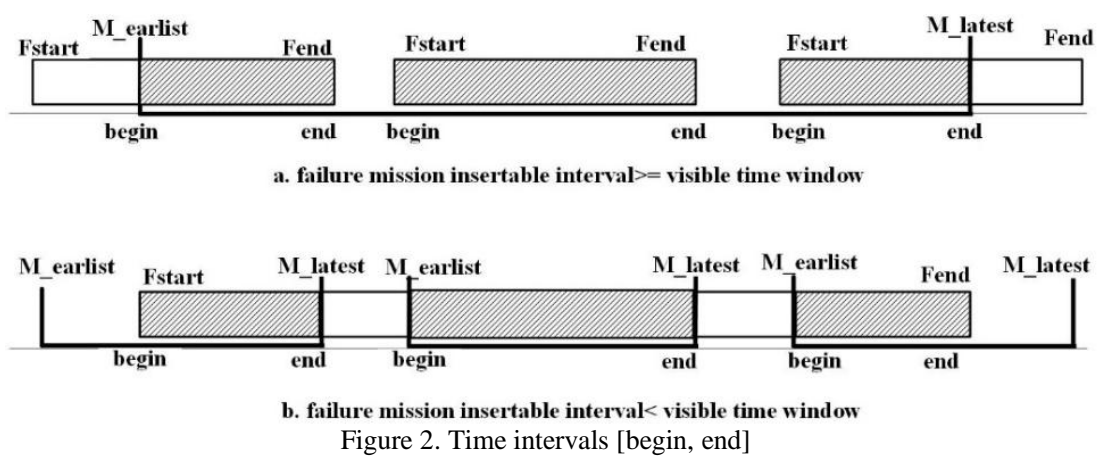

\subsection{User Case Simulation and Result Analysis}

The scenario in the user case consists of five satellites, three ground stations, and two relay satellites. Each station has one antenna whose service type is a function integrated antenna. The mission group of each satellite and the fault parameters of each antenna are shown in Tables 2 and 3, respectively. The simulation results are obtained on a PC with a 2.6-GHz Intel Core i7 CPU and 8Gbytes RAM on the Windows 10 operating system. The simulation time is one week, and the simulation step is five minutes. The resource scheduling simulation is executed ten times, and the reliability simulation is executed one hundred times independently.

Table 2. Mission groups of each satellite

\begin{tabular}{|c|c|c|c|c|c|c|c|c|c|}
\hline \multicolumn{1}{|c|}{ Satellite } & \multicolumn{7}{|c|}{ Mission ID } \\
\hline name & priority & $a$ & $b$ & $c$ & $d$ & $e$ & $f$ & $g$ & $h$ \\
\hline Satellite1 & 6 & 3 & 3 & 3 & 3 & 3 & 3 & 15 & 15 \\
\hline Satelite2 & 9 & 3 & 3 & 3 & 3 & 3 & 3 & 15 & 15 \\
\hline Satelite3 & 5 & 3 & 3 & 3 & 3 & 3 & 3 & 15 & 15 \\
\hline Satelite4 & 6 & 3 & 3 & 3 & 3 & 3 & 3 & 15 & 15 \\
\hline Satelite5 & 6 & 3 & 3 & 3 & 3 & 3 & 3 & 15 & 15 \\
\hline
\end{tabular}

Table 3. Fault parameters of each antenna

\begin{tabular}{|c|c|c|c|}
\hline Antenna name & Station & MTBF & MTTR \\
\hline G_Transmitter1 & Ground1 & 20 & 10 \\
\hline G_Transmitter2 & Ground2 & 25 & 10 \\
\hline G_Transmitter3 & Ground3 & 41 & 10 \\
\hline RelayTransmitter1 & relay1 & 28 & 10 \\
\hline RelayTransmitter2 & relay2 & 35 & 10 \\
\hline
\end{tabular}

The reliability simulation results of the satellite 1 mission group are given in three cases: system without failure, system with failure, and system with failure mission rescheduling, as shown in Figure 3. Compared with the system mission completion rate without rescheduling, the system has improved the rate after considering the rescheduling method to these failure missions with dynamic redundancy. It means that the system has higher mission reliability after considering the system dynamic redundancy.

\section{Conclusions}

Considering that the existing analytical methods are insufficient to evaluate the reliability of system missions with dynamic redundancy, this paper designed a discrete event simulation method combined with Monto Carlo theory to obtain an approximate solution. The simulation process was divided into two parts: the first part generated the feasible scheduling 


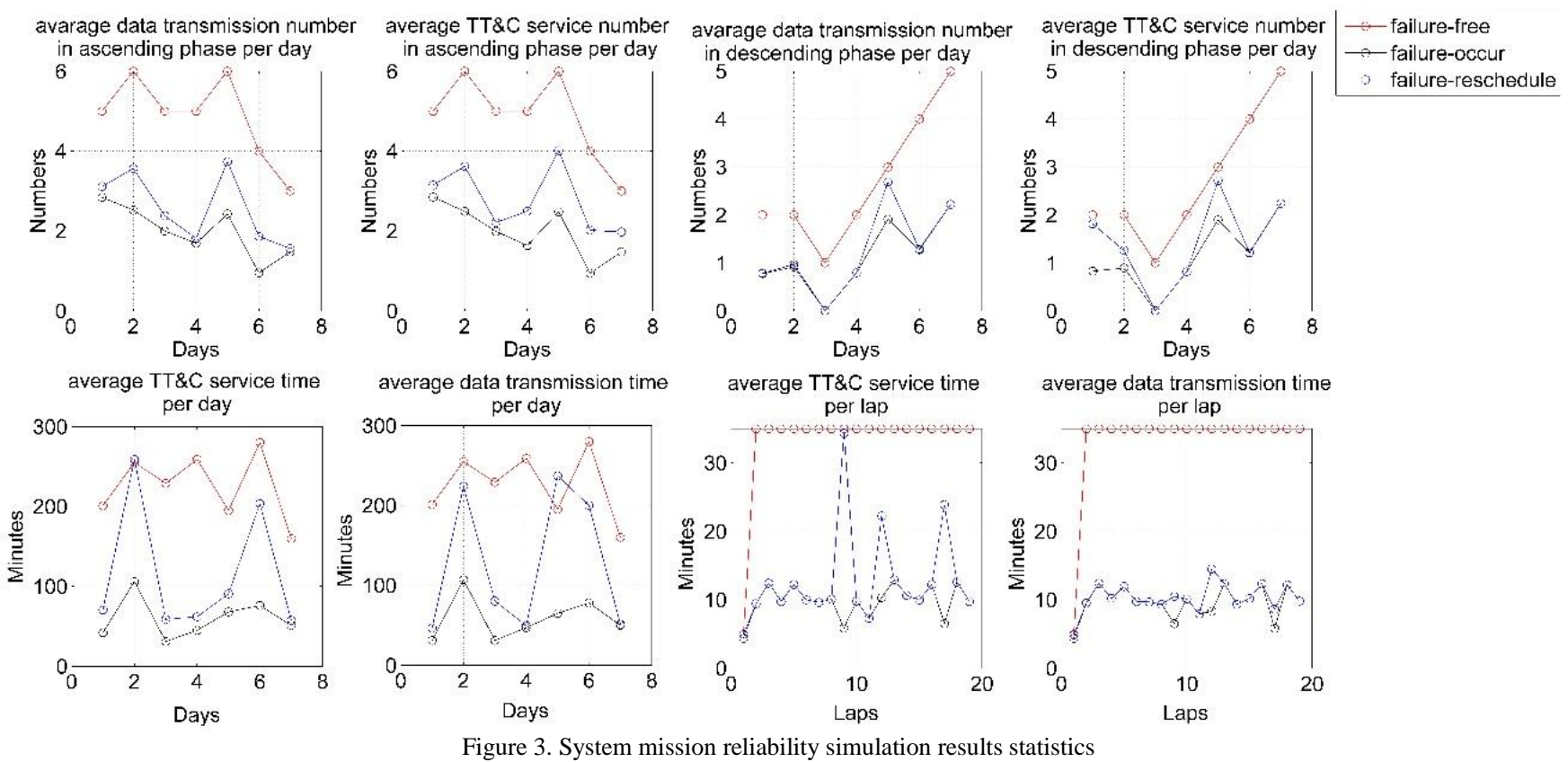

plans through the resource heuristic scheduling algorithm, while the second part simulated the mission reliability of the system with the failure mission rescheduling algorithm. The simulation results statistics of the user case showed that the system mission reliability was improved after considering the system dynamic redundancy.

The work performed in this paper only marks the beginning for system mission reliability with dynamic redundancy. The rescheduling algorithm chose unoccupied time periods for failure mission insertion. However, most antennas are occupied in the schedule plan due to a large number of missions in the actual scheduling process, and the rescheduling algorithm must deal with resource conflicts in this case. Discrete event simulation is very intuitive for resource scheduling processes with high time consumption, and the result is not accurate. Thus, future studies will focus on extending the traditional analytical method to analyze the system mission reliability with dynamic redundancy.

\section{Acknowledgments}

This work is partially supported by the National Natural Science Foundation of China (No. 71671185).

\section{References}

1. Q. Dang, "Designed on Operation and Management System for Aerospace TT\&C Station," in Proceedings of the $28^{\text {th }}$ Conference of Spacecraft TT\&C Technology in China, pp. 579-590, 2016

2. J. Liu, "Spacecraft TT\&C and Communication Engineering," National Defense Industry Press, 2010

3. K. M. Aron, G. Fox, and D. H. Ebbeler, "Reliability Modeling for the Space Interferometry Mission," in Proceedings of IEEE Aerospace Conference, Vol. 2, pp. 689-704, 2002

4. F. Hamed and S. T. A. Niaki, "Fault Tree Analysis for Reliability Evaluation of an Advanced Complex Manufacturing System," Journal of Advanced Manufacturing Systems, Vol. 17, No. 1, pp. 107-118, 2018

5. L. Anatoly, "A Multi-State Markov Model for a Short-Term Reliability Analysis of a Power Generating Unit," Reliability Engineering \& System Safety, Vol. 98, No. 1, pp. 1-6, 2017

6. H. Xie, E. Gong, and Y. He, "Study on Reliability of Phased Missions System with Time-Varying Structure," Journal of National University of Defense Technology, Vol. 21, No. 5, pp. 41-45, 1999

7. X. Wu, G. Wang, and L. Lu, "An Approach to Mission Reliability Analysis of Spaceflight TT\&C System," in Proceedings of the 4th Asia-Pacific International Symposium on Advanced Reliability Modeling, pp. 748-755, 2010

8. X. Yang and X. Wu, "Mission Reliability Assessment of Space TT\&C System by Discrete Event System Simulation," Quality \& Reliability Engineering International, Vol. 30, No. 8, pp. 1263-1273, 2015

9. S. Xu, "A Simulation Method for Space TT\&C Communication System Mission Reliability Evaluation," 'National University of Defense Technology, 2013

10. X. Wu and J. Hillston, "Monte Carlo Simulation for Reliability Estimation of Phased-Mission Systems With Minimum Operational Time Requirement," in Proceedings of the 6th International Conference on Quality, Reliability, Risk, Maintenance, and Safety Engineering, Jiuzhaigou, China, July 2016

11. J. Xiong, R. Leus, and Z. Yang, "Evolutionary Multi-Objective Resource Allocation and Scheduling in the Chinese Navigation 
Satellite System Project," European Journal of Operational Research, Vol. 251, No. 2, pp. 662-675, 2016

12. W. Herroelen and R. Leus, "Project Scheduling under Uncertainty: Survey and Research Potentials," European Journal of Operational Research, Vol. 165, No. 2, pp. 289-306, 2005

Haiyue $\mathbf{Y u}$ is a Ph.D. student in management science and engineering in the School of System Engineering at National University of Defense Technology. His research interests are system evaluation and decision analysis.

Xiaoyue Wu is a professor in management science and engineering in the School of System Engineering at National University of Defense Technology. His main research interests include reliability modeling, evaluation of complex systems, system tests and evaluation, and decision analysis under uncertainty. He has been a principal investigator for more than ten system reliability-related research projects, and he has won six awards for his research contributions in the field. 